\title{
Analysis of driving properties of a three-wheeled vehicle with a newly designed steering system
}

\author{
Ján Dižo ${ }^{1, *}$, Miroslav Blatnický ${ }^{1}$, and Pavol Kurčík ${ }^{1}$ \\ ${ }^{1}$ Department of Transport and Handling Machines, Faculty of Mechanical Engineering, University of \\ Žilina, Univerzitná 8215/1, 01026 Žilina, Slovak Republic
}

\begin{abstract}
This paper presents results of analyses of a three-wheeled vehicle with the newly designed steering system. Our design of steering system is intended to be mounted on a tricycle with the electric drivetrain. The standard steering system of a three-wheeled vehicle has disadvantage, that such a vehicle is unstable, when it is curving a curve mainly at higher speeds. It results to a breach of stability form the overturning point of view. The newly designed steering system of a three-wheeled vehicle contributes to increasing its stability. It means that a three-wheeled vehicle with the newly designed steering system is able to pass a curve with a smaller radius at higher speed. The main objectives of analyses presented in this paper is comparison of driving properties of a three-wheeled vehicle with two wheels on a rear axle with the standard steering system and with the newly designed steering system by means of simulation computations, which were carried out in Simpack package.
\end{abstract}

Keywords: three-wheeled vehicle, driving properties, overturning stability, simulation computations

\section{Introduction}

Current consumption of fossil fuels of motor vehicles and the trend of prices related with it with forces car companies to develop more fuel efficient passenger vehicles. At present, standard four-wheeled vehicles are designed to accommodate four to six passengers comfortably with sufficient space for their luggage. This design creation limits significant improvements in the fuel efficiency of these vehicles. On the other hand, two-wheeled vehicles such as bicycles, mopeds and motorcycles are extremely fuel efficient. However, these two-wheeled vehicles are primary designed as single passenger vehicles with limited luggage compartment. In addition, they do not protect enough passengers from bad weather conditions on desired form. This makes them unsatisfactory for the majority for the driving public. Therefore, there is a need for design of such vehicles which will be able to use for transportation of one or two passengers with some of the space and comfort afforded by standard four-wheeled vehicles and significantly greater fuel efficiency [1,2].

\footnotetext{
${ }^{*}$ Corresponding author: jan.dizo@,fstroj.uniza.sk

Reviewers: Tomáš Lack, Konrad Waluś
} 
Recently engineers of transport means make a considerable effort to think up effective way to save fossil fuel not only for road vehicles [3], but also for rail vehicles and others. There are many research focused on fuels saving, such as using alternative fuels $[4,5]$, combination of several sources of energy know as hybrid drivetrains [5-8], nay to make use of same phenomena, e. g. waste heat during braking etc $[9,10]$. Some of these approaches are more effective and but some of them still meet associated problems, such as negative effects from the durability point of view, operating behaviours etc [11, 12].

Application of an electric drivetrain for lower-category urban car is one of the most effective ways for reducing fossil fuels consumption and exhaust production [13]. Electric motor drive of vehicles is already applied in several areas of passenger transport $[1,14]$ and also three-wheeled vehicles popularize. These facts result to various design of lower-category urban cars with three wheels.

\section{Fundamentals of the technical solution}

Basically there are two configurations of three-wheeled vehicles namely with two wheels on a rear axle and with two wheels on a front axle. As usual, both dispose of advantages and also disadvantages. In our project of a design of a lower-category urban vehicle with electric drivetrain we have decided to become attached to the conception of a three-wheeled vehicle with two wheels on a rear axle. The essential disadvantage of such a wheels layout is the worse overturning stability of a vehicle when it is passing curves. It becomes evident mainly in curves with small radius at higher speeds. Therefore in our workplace the task to solve this problem has risen. The effort has eventuated to the new technical solution of the steering system of a three-wheeled vehicle [15].

Hence the goal of this contribution is to present the comparison of driving properties of three-wheeled vehicles with two wheels on a rear axle, at which one vehicle contains the standard steering system and the other is equipped with the newly designed steering system. There was monitored the case of curve passing. Analyses were carried out in Simpack software [15].

A technical solution relates to the front wheel fork design of an electric tricycle with two wheels on the rear axle. The goal is to improve the overturning stability of such a vehicle during driving in curves.

Nowadays, steering mechanisms of three-wheeled vehicles with two wheels on the rear axle use a standard fork for the front wheel mounted on a vehicle chassis.

In current solutions of the standard tricycle steering, the contact point between the front wheel and the road during driving in curves is changeless. Then, the arising centrifugal force is compensated by passengers tilting in the opposite direction, because the centre of gravity is near to the stability axis, which is given by the jumper lead of the front wheel and the external rear wheel in a curve.

Therefore, there has been occurred the requirement for the design of such front wheel suspension structure, which would ensure the overturning stability improvement of an electric tricycle and besides the conditions of the stability during braking has to be satisfied. The result of this effort is the new design of the front fork suspension of an electric tricycle.

The fundamental of our technical solution is the fact, that the front part of an electric tricycle contains the combination of rotating and shifting joints, which allows in addition to the rotary movement also the shifting movement in the lateral direction [15].

Comparison of the fundamentals of the difference between the standard steering system of an electric tricycle and the new designed steering system is shown in Fig. 1, where virtual models of both created by means of Simpack multibody package are presented.

Advantages of the technical solution of the front wheel fork suspension of an electric tricycle are apparent from its effects, by which it expresses itself outwards. Effects of this 
technical solution are in that this design allows overturning stability improvement of an electric tricycle, which uses a steering of the motorcycle type. The introduced design is based on the front wheel shifting to the right side during driving in the left curve (Fig. 1) and contrariwise, i.e. always in the centrifugal force direction in order to improve the overturning stability.

a)
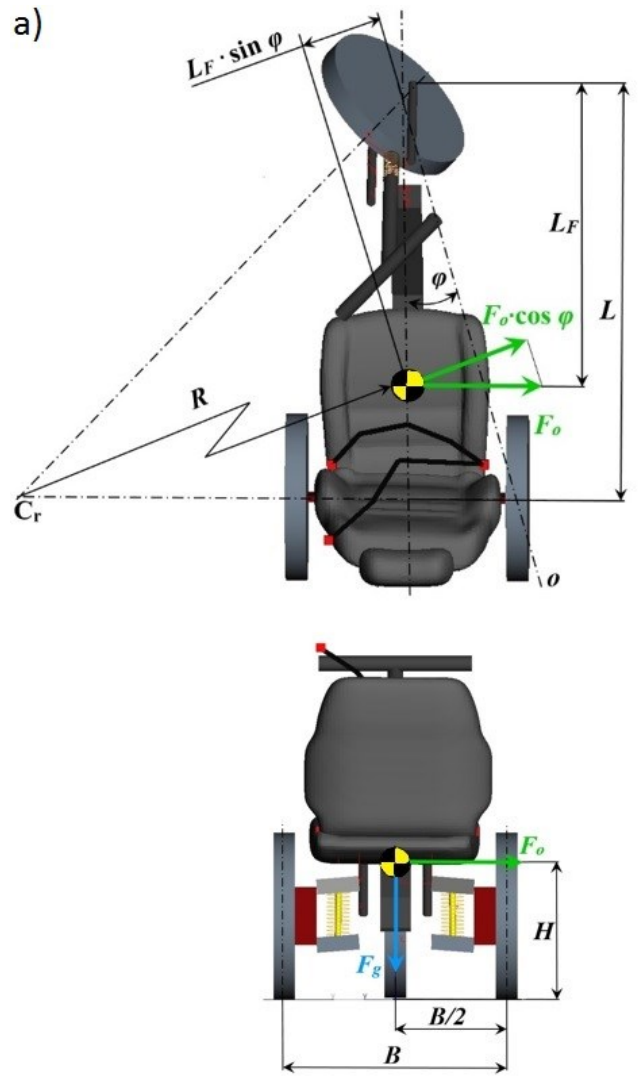

b)

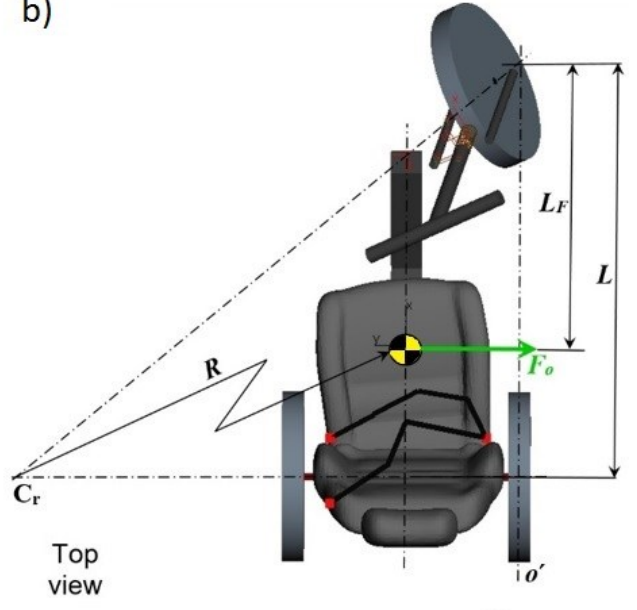

Rear view

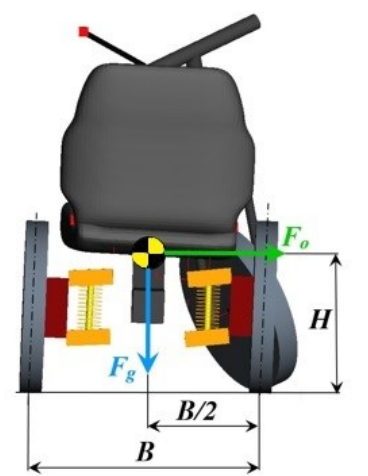

Fig. 1. Curved track run of the three-wheeled vehicle with the standard steering system (a) and with the newly designed steering system (b)

\subsection{Vehicle overturning stability in curves}

This section contains geometrical relationships, which are related to the overturning stability of a three-wheeled vehicle with two wheels on the rear axle, when it is driving in a curve. The overturning stability depends on the lateral acceleration, which rises therein. It is assumed, that this lateral acceleration acts perpendicular to the centre line of a vehicle. There are compared two technical solutions, a three-wheeled vehicle with the standard steering system and with the new designed steering system, which allows except for the inevitable rotation also the lateral movement of the front fork with a wheel [16, 17].

In Fig. 1 the top views and rear views of both configurations of a three-wheeled vehicle with two wheels on the rear axle are shown. A three-wheeled vehicle with the standard steering system is located and a three-wheeled vehicle with the new technical solution of the front fork is found on the right. The $o$ axis represents the tipping axis. To ensure the overturning stability, the moment reacting in the negative direction about $o$ axis, must exist (see the rear view of the vehicle). 
Firstly, we analyse the overturning stability of the standard three-wheeled vehicle model, which is shown in Fig. 1a. The sum of moments about the $o$ axis is given by the following equation:

$$
\sum_{i} M_{i o} \leq 0 \Rightarrow F_{o} \cdot \cos \varphi \cdot H-F_{g} \cdot L_{F} \cdot \sin \varphi \leq 0,
$$

where $F_{o}[\mathrm{~N}]$ is force, which causes a lateral acceleration (centrifugal force), $H[\mathrm{~m}]$ is the assumed height of the centre of gravity of a vehicle (including a driver), $L_{F}[\mathrm{~m}]$ symbolize the position of the centre of gravity from the front wheel and $\varphi\left[^{\circ}\right]$ is the angle given by the vehicle design. From eq. 1, we can derivate the formula, which represents the overturning stability of a standard three-wheeled vehicle:

$$
\frac{a_{o}}{g} \leq \frac{l_{F}}{h} \cdot \tan \varphi
$$

where $g\left[\mathrm{~m} \cdot \mathrm{s}^{-2}\right]$ is the gravity acceleration. The $\varphi$ angle is a function of the vehicle design and we can express it following:

$$
\tan \varphi=\frac{B}{2 \cdot L},
$$

where $B[\mathrm{~m}]$ is rear wheels base and $L[\mathrm{~m}]$ is the axle base.

If we substitute eq. 3 into eq. 2 , the condition of the overturning stability of a threewheeled vehicle with standard steering system and with two wheels in the rear axle is:

$$
\frac{a_{o}}{g} \leq \frac{B}{2 \cdot H} \cdot \frac{L_{F}}{L} .
$$

Let's compare just derived formulas with those, which describe the overturning stability of a three-wheeled vehicle with the newly designed steering system. As it enables the lateral movement of the front wheel, we will consider a case in compliance with Fig. 1b. Thereafter the sum of moments about the $o^{\prime}$ axis is:

$$
\sum_{i} M_{i O^{\prime}} \leq 0 \Rightarrow F_{o} \cdot h-F_{g} \cdot \frac{B}{2} \leq 0
$$

Analogous to the previous case, it can be rewritten as:

$$
\frac{a_{o}}{g} \leq \frac{B}{2 \cdot h}
$$

Now we take into account some physical feeling. When these vehicles are driving in a curve with the radius $R$, in the centre of gravity of both the corresponding lateral acceleration is rising. The calculation of its value in dependence on a vehicle forward speeds $v\left[\mathrm{~m}_{\mathrm{s}} \mathrm{s}^{-1}\right]$ and a curve radius $R[\mathrm{~m}]$ is given by the known relation:

$$
a_{o}=\frac{v^{2}}{R},
$$

where $a_{o}\left[\mathrm{~m} \cdot \mathrm{s}^{-2}\right]$ is the lateral acceleration. Substituting eq. 7 into relations 4 and 6 we obtain formulations for the maximum forward speed $v_{\max }$ and $v^{\prime}{ }_{\max }$ of a three-wheeled vehicle with the standard steering system and with the newly designed steering system, respectively, at 
which the overturning comes into being. Hence, the formula for the overturning speed for the standard steering system is:

$$
v_{\max } \leq \sqrt{\frac{B}{2 \cdot h} \cdot \frac{L_{F}}{L} \cdot R \cdot g},
$$

and for the overturning speed for the newly designed steering system:

$$
v_{\max }^{\prime} \leq \sqrt{\frac{B}{2 \cdot h} \cdot R \cdot g} .
$$

In balance, the expression 9 for the speed represents the maximum driving speed of a fourwheeled vehicle.

If we take into account main dimensions of vehicles and compare equations 8 and 9 , we can see that a value of the dimension is anyway smaller than the axle base $L$. It appears from this that wheel the overturning stability of a three-wheeled vehicle with the standard steering system is worse than the overturning stability of a three-wheeled vehicle it the newly designed steering system provided corresponding parameters.

\section{Comparison and assessment of driving properties of three- wheeled vehicles driving in a curve}

Hence, there are presented results of dynamic analyses of the driving two three-wheeled vehicles, namely the vehicle with the standard steering system and with the newly designed steering system. Assessment and comparison of the driving properties of both is performed based on simulation computations carried out in Simpack software package. Moreover, we have also compared results of these numerical calculations with the analytical approach. The used software enables modelling and analysing of the dynamic properties and behaviour of transport means of wide range inclusive road vehicles, rail vehicles [18-20] and similar transport means ranging from trams [21-23] to high-speed trains. For calculations of contact phenomena between wheel (tire or railway wheel) and track (road or railway track) it used variously intricate models [24, 25]. These elements of any multibody vehicle model represent at once an inseparable and a quite difficult modelling problem [26-28].

In order to achieve such a virtual model, which represent as faithfully as possible the reality, we have created three-dimensional models of individual parts of both three-wheeled vehicles versions. Since we found out mass and inertia parameters including locations of centres of gravity we were subsequently input them into the vehicles multibody models [2931]. Moreover we have also considered a driver with standardized parameters sitting on a seat. Once we have set up complete vehicles multibody models, we detected the total mass and inertia parameters of them and also total centres of gravity, which are necessary input for analytical calculations.

As noted previously, we have focused our interest on the fact, whether and how our newly designed steering system is able to improve the overturning stability of a three-wheeled vehicle with three wheels on the rear axle. For this purpose we have chosen the driving manoeuvres in a curve with relatively the radius of $R=2.0 \mathrm{~m}$. Subsequently we performed numerical simulations at various speeds and we investigated the moment, when the overturning stability of both vehicles is breached. As the evaluative criterion we have determined the contact force between the internal rear wheel in a curve and the road. The extreme case is, when the contact force equals zero. 
From relations derived above (equations 8 and 9), from the view of vehicles parameters the overturning stability criteria for both the standard steering system and the newly designed steering system do not depend on the mass of vehicles, but only on positions of centres of gravity in the longitudinal and lateral direction and on main construction parameters of vehicles, i. e. axle base and rear wheels base. Based on derived and determined we can analyse the maximum forward speed of both vehicles.

Let's take into account parameters of a three-wheeled vehicle resulting from its design. The axle base is $L=1.317 \mathrm{~m}$ and the rear wheels base is $B=0.727 \mathrm{~m}$. The position of the centre of gravity is given by two parameters, namely by its height $H=0.43 \mathrm{~m}$ and its position from the front wheel $L_{F}=0.935 \mathrm{~m}$. We take an interest, what the maximum forward speed the three-wheeled vehicle with the standard steering system can driving the curve with the radius $R=2.0 \mathrm{~m}$ at so as not to breach the overturning stability. If we substitute these parameters into eq. 8 , we get:

$$
v_{\max } \leq \sqrt{2.0 \cdot 9.81 \cdot \frac{0.935}{1.317} \cdot \frac{0.727}{2 \cdot 0.43}},
$$

and the calculated value is:

$$
v_{\max } \leq 3.887\left[\mathrm{~m} \cdot \mathrm{s}^{-1}\right]=13.993\left[\mathrm{~km} \cdot \mathrm{h}^{-1}\right] .
$$

In Figs. 2 and 3 results from numerical analyses are shown. They contain results of driving the three-wheeled vehicle with the standard steering system (Fig. 2) and the three-wheeled vehicle with the newly designed steering system in the curve and additional illustrations from animations of vehicles driving. There are depicted waveforms of values of the total vertical wheel forces in the contact of the internal rear wheel and the road, as noted previously with numerical values of these parameters.

Now, we can compare result from the analytical calculation (eq. (11)) with results from simulation computations. It is possible to detect, that the limited value of the forward speed of the three-wheeled vehicle with the standard steering system is really proper found out. The little difference is caused by the fact that during the vehicle driving in a curve a passenger is shifting in the direction of a lateral acceleration and therefore the centre of gravity is also shifting a bit in this direction.

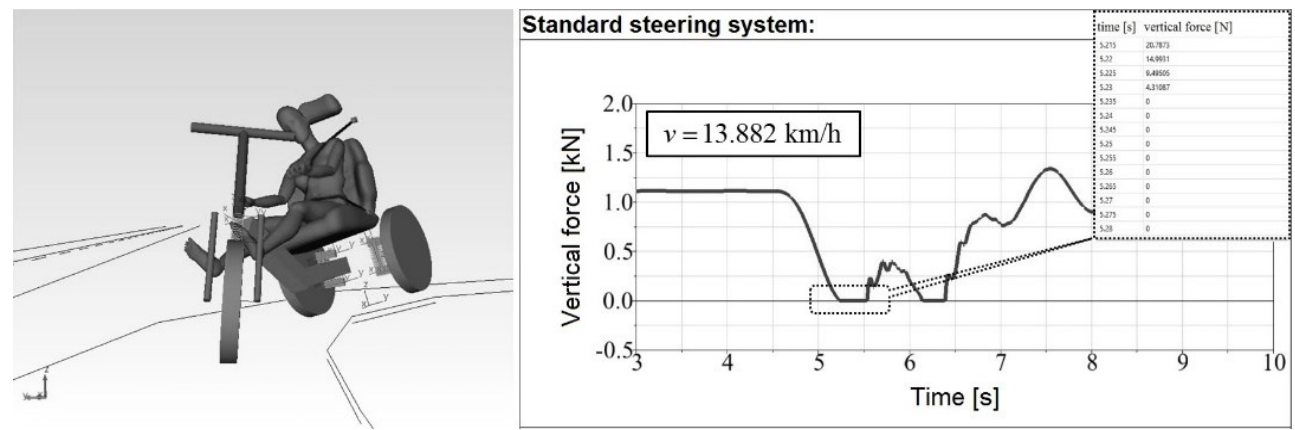

Fig. 2. Results of numerical analyses of the electric tricycle with the standard steering system 


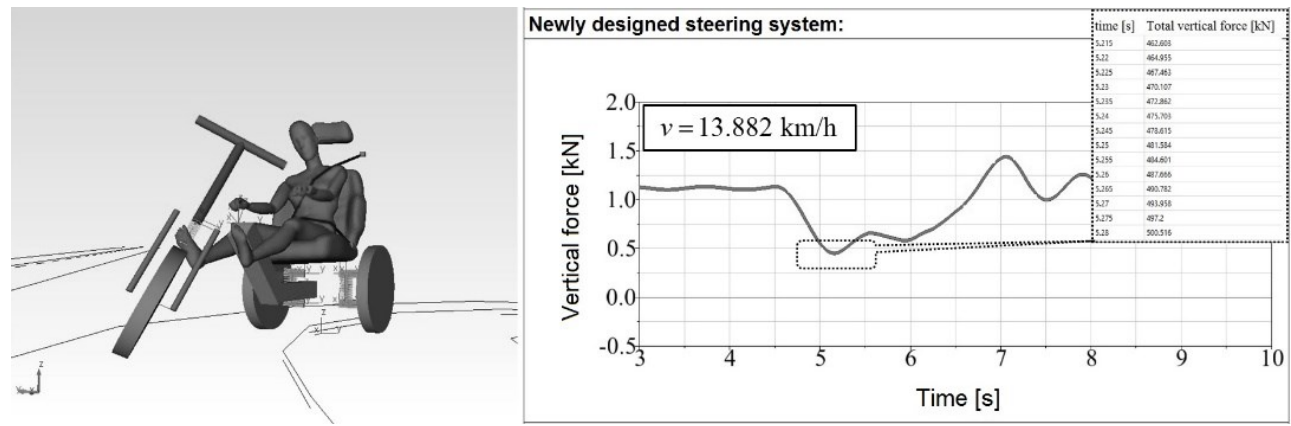

Fig. 3. Results of numerical analyses of the electric tricycle with the new designed steering system

Next, from results of simulation computations the three-wheeled vehicle with the newly designed steering system we can observe (Fig. 3) that this innovative technical solution improves in reality the overturning stability of a three-wheeled vehicle with two wheels on the rear axle.

It is clearly proven by performed calculations. Values of the rear internal wheel forces during the entire cornering are over zero values. It means the internal rear wheel does not lose contact with the road at all, and due to the parameters of the three-wheeled vehicle and driving conditions, contact forces on the internal rear wheel have sufficient values throughout entire cornering to ensure the satisfactory overturning stability of a vehicle.

Based on performed calculation and analyses, both analytical and numerical, we have found out that using our newly designed steering system for the design of a three-wheeled vehicle with two wheels on the rear axle really facilitates to improve the overturning stability of such a three-wheeled vehicle.

\section{Future research activities}

To summarize there is necessary to remark several important findings and facts about contents. This technical solution represents a primary prototype of a three-wheeled vehicle with our designed steering system [32]. Let's come back to Fig. 1, where the keynote of improvement of the overturning stability of a three-wheeled vehicle is presented. The odds are that in a real vehicle with three wheels mounted in considered configuration there will not be possible to achieve such the significant lateral shifting of the front wheel as in the ideal scheme. There is because of several reasons.

If we take a think about the maximal effectiveness of the mechanism, then it would has to shift the front wheel in the lateral direction during driving at any speed in a curve to the maximal position, i. e. to a half of the rear wheel gauge. Thus, it would mean, that the mechanism would be controlled by a device, which would be able to identify actual speed of the vehicle and to adapt lateral shifting of the wheel. This modification of the mechanism would be much difficult in comparison with considered fully mechanical system, but also other considerable adverse fact, which would arise during driving at low speeds. If we consider the maximal lateral shifting of the front wheel, such conditions would necessarily come into being, when the vehicle would come into the unstable state and it would overturn inside a curve [32].

Another technical problem would be with the proper steering-system geometry setting. At the significant lateral shifting, the front wheel is very inclined. It is related not only with massive tyre wear but mainly with the changing of load of individual parts of the steering system. 
Therefore in our workplace such a prototype was produced, which uses only mechanical steering system, which enables the partial improvement of the overturning stability by the lateral shifting of the front wheel, whereby the load of parts of steering mechanism is remained on the acceptable limits.

Future research in this field will be concerned with a modification of our prototype and with a development this technical solution in such a manner, which will be acceptable on the one hand from the utility point of view and on the other hand from the point of view of its simple application for the common use. In addition we want to develop this steering system moreover that it will be able to apply not for electric three-wheeled vehicle but for vehicles with any kind of a drivetrain.

\section{Conclusion}

The new idea of a new technical solution of a steering system of three-wheeled vehicles with two wheels on the rear axle was presented. As a usage of electric drivetrain is currently one of the key factors of reducing of the consumption of fossil fuel, the new design of the steering system is intended to be mounted on an electric three-wheeled vehicle in order to improve same driving characteristics, mainly the overturning stability such a vehicle when it is driving in curves. It is very closely related to the passenger's safety. This technical solution is interesting by working purely on the mechanical principle.

From performed calculation and analyses we found out, that the newly designed steering system really improves the overturning stability of a three-wheeled vehicle. We have proved it by means of results from simulation computations of a cornering two versions of a vehicle, i. e. with the standard steering system and with the newly designed steering system at the same driving conditions (speed, curve radius).

The work was supported by the Cultural and Educational Grant Agency of the Ministry of Education of the Slovak Republic in project No. KEGA 077ŽU-4/2017: Modernization of the Vehicles and engines study program. This work was supported by the Grant program of the Volkswagen Slovakia Foundation in the project: E-3kolka.

\section{References}

1. T. Skrucany, M. Kendra, M. Skorupa, M., J. Grencik, T. Figlus, Comparison of chosen environmental aspects in individual road transport and railway passenger transport. Procedia Engineering 192, 806-811 (2017)

2. M. Mruzek, I. Gajdac, L. Kucera, D. Barta, Analysis of parameters influencing electric vehicle range. Procedia Engineering 134, 165-174 (2016)

3. P. Baran, M. Brezani, P. Kukuca, P. Stastniak, Basic dynamical analysis and comparison of balancing systems of non-conventional piston machine FIK. Procedia Engineering 192, 34-39 (2017)

4. J. Caban, A. Gniecka, L. Holesa, Alternative fuels for diesel engines. Advances in science and technology - research journal 7, 70-74 (2013)

5. D. Barta, M. Mruzek, M. Kendra, P. Kordos, L. Krzywonos, Using of non-conventional fuels in hybrid vehicle drives. Advances in science and technology-research journal 10, 240-247 (2016)

6. A. Falendysh, M. Volodarets, O. Kletska, V. Hatchenko, The impact of the type of operation on the parameters of a shunting diesel locomotive with hybrid power plant. MATEC Web of Conferences, 133 (2017) 
7. A. Falendysh, P. Kharmalov, O. Kletska, N. Volodarets, Calculation of the parameters of hybrid shunting locomotive. Transport research arena TRA2016 14, 665-671 (2016)

8. P. Drozdziel, I. Rybicka, R. Madlenak, A. Andrusiuk, D. Siluch, The engine set damage assessment in the public transport vehicles. Advances in science and technology research journal 11, 117-127 (2017)

9. A. Suchanek, J. Harusinec, M. Loulova, P. Strazovec, Analysis of the distribution of temperature fields in the braked railway wheel. MATEC Web of conferences, 157 (2018)

10. A. Suchanek, J. Harusinec, The downhill braked railway wheel structural analysis my menas of the ANSYS Multiphysics program system package. Manufacturing Technology 15, 945-950 (2015)

11. G. Vaiciunas, G. Bureika, S. Steisunas, Research on metal fatigue rail vehicle wheel considering the wear intensity of rolling surface. Eksploatacja i Niezawodnosc Maintenance and Reliability 20, 24-229 (2018)

12. L. Smetanka, P. Stastniak, J. Harusinec, Wear research of railway wheelset profile by using computer simulation. MATEC Web of Conferences, 157 (2018)

13. B. Sarkan, O. Stopka, J. Gnap, J. Caban, Investigation of exhaust emissions of vehicles with the spark ignition engine within emission control. Procedia Engineering 187, 775782 (2017)

14. G. Bureika, S. Steisunas, Complex evaluation of electric rail transport implementation in Vilnius city. Transport Problems 11, 49-60 (2016)

15. J. Dizo, D. Barta, M. Blatnicky, Improvement driving characteristics of electric tricycle. Engineering for Rural Development 2018 17, 2123-2128 (2018)

16. J. A. Jubin, K. Jaybal, Rollover dynamics of a narrow tilting three-wheeled vehicle. MATEC Web of Conferences 51 (2016)

17. J. Barote, J. Darling, A. Plummer, Lateral dynamics simulation of a three-wheeled tilting vehicle. Proceedings of the Institution of mechanical Engineers, Part D: Journal of Automobile Engineering 229, 342-356 (2015)

18. R. Melnik, B. Sowinski, Analysis of dynamics of a metro vehicle model with differential wheelsets. Transport Problems 12, 113-124 (2017)

19. M. Kostrzewski, R. Melnik, Numerical dynamics study of a rail vehicle with differential gears. Procedia Engineering 192, 439-444 (2017).

20. M. Kostrzewski, Implementation of distribution model of an international company with use of simulation method. Procedia Engineering 192, 445-450 (2017)

21. V. Hauser, O. Nozhenko, K. Kravchenko, M. Loulova, J. Gerlici, T. Lack, Car bod and bogie connection modification for track curves passability improvement. MATEC Web of Conferences 157 (2018)

22. V. Hauser, Nozhenko, O., Kravchenko, K., Loulova, M., Gerlici, J., Lack, T. Proposal of a steering mechanism for tram bogie with three axle boxes. Procedia Engineering 192, 289-294 (2017)

23. V. Hauser, O. Nozhenko, K. Kravchenko, M. Loulova, J. Gerlici, T. Lack, T. Impact of three axle boxes bogie to the tram behaviour when passing curved track. Procedia Engineering 192, 295-300 (2017)

24. J. Gerlici, T. Lack, Contact geometry influence on the rail/wheel surface stress distribution. Procedia Engineering 2, 2249-2257 (2010)

25. J. Gerlici, T. Lack, Modified HHT method for vehicle vibration analysis in time domain utilisation. Applied mechanics and Materials 486, 396-405 (2014) 
26. T. Lack, J. Gerlici, A modified strip method to speed up the calculation of normal stress between wheel and rail. Applied Mechanics and Materials 486, 359-370 (2014)

27. T. Lack, J. Gerlici, A modified strip method to speed up the tangential stress between wheel and rail calculation. Applied Mechanics and Materials 486, 371-378 (2014)

28. J. Gerlici, T. Lack, J. Harusinec, Realistic simulation of railway operation on the RAILBCOT test stand. Applied Mechanics and Materials 486, 387-395 (2014)

29. A. Sapietova, J. Bukovan, M. Sapieta, L. Jakubovicova, Analysis and implementation of input load effects on an air compressor piston in MSC.ADAMS. Procedia Engineering 177, 554-561 (2017)

30. M. Saga, L. Jakubovicova, Simulation of vertical vehicle non-stationary random vibrations considering various speeds. Scientific journal of Silesian University of Technology 84, 113-118 (2014)

31. A. Sapietova, V. Dekys, M. Sapieta, P. Pechac, Application of computational and design approaches to improve carries stability. Procedia Engineering 96, 410-418 (2014)

32. P. Strazovec, P. Kurcik, A. Pavlik, M. Blanticky, E3-kolka with a mechanism for improvement of stability when passing through the curve (In Slovak). Proceedings of $5^{\text {th }}$ conference for young scientific workers Experimental and computational methods in engineering, J. E. Purkyne University in Usti nad Labem, June 6-8, 2018. 